\title{
Image-guided Femtosecond Laser Capsular Marks for Toric Intraocular Lens Alignment-The Refractive Capsulorhexis
}

\author{
Mark Packer \\ Mark Packer MD Consulting, Inc. Boulder, CO, USA
}

$\mathrm{T}$ he correction of astigmatism is fundamental to the performance of refractive cataract surgery. Important modalities for the correction of astigmatism include corneal arcuate incisions and toric intraocular lens (IOL) implantation. In regard to toric lens implantation, correct alignment of the lens with regard to the axis of astigmatism constitutes a critical element of the procedure. Various methods have been developed for accurate toric lens alignment. In this paper we review preclinical and clinical results of a novel method for toric lens alignment: the construction of image-guided femtosecond laser capsular marks. Laboratory data show that the introduction of these marks does not negatively impact the strength or extensibility of the capsulotomy, while clinical data suggest that the use of capsular marks for toric lens alignment has the ability to significantly enhance refractive outcomes. Accurate alignment of toric IOLs represents a critical step in the reduction of preexisting corneal astigmatism for patients desiring spectacle independence following cataract surgery. The demonstrated safety and effectiveness of IntelliAxis Refractive Capsulorhexis ${ }^{\mathrm{TM}}$ (LENSAR ${ }^{\circledR}$, Orlando, FL, USA) provides the clinical foundation for improved postoperative results and enhanced patient satisfaction.

\section{Keywords}

Astigmatism, toric intraocular lens,

femtosecond laser-assisted cataract surgery

Disclosure: Mark Packer serves as a consultant to Advanced Vision Science, Inc. (Santen), Alcon Research, Bausch Health, EarlyBird Health-Tech Management, Keranova, LENSAR (PDL, Inc.), Olympic Ophthalmics, Inc. Promedica International, Precision for Medicine, Inc., Rayner Intraocular Lenses Ltd., SOLX, Inc., and University of Colorado; as a consultant to and an investor in Cassini Technologies B.V., ClearSight, International BioMedical Devices, Inc., Ira, Inc., LensGen, Inc., Refocus Group, Inc., STAAR Surgical Co., Tarsus Pharmaceuticals, Inc., and Visant Medical, Inc.; and as an investor in Aerie Pharmaceuticals, lantech, Inc., Transcend Medical, Inc., TrueVision Systems, Inc., and WaveTec Vision Systems, Inc.

Review Process: Double-blind peer review.

Compliance with Ethics: This study involves a review of the literature and did not involve any studies with human or animal subjects performed by any of the authors.

Authorship: The named author meets the International Committee of Medical Journal Editors (ICMJE) criteria for authorship of this manuscript, takes responsibility for the integrity of the work as a whole, and has given final approval for the version to be published.

Received: August 15, 2019

Accepted: October 14, 2019

Citation: US Ophthalmic Review. 2019;12(2)60-4

Corresponding Author: Mark Packer, Mark Packer MD Consulting, Inc. 1400 Bluebell Ave. Boulder, CO 80302, USA. E: mark@markpackerconsulting.com

Support: The production of this paper was supported in part by LENSAR (PDL, Inc.).
Approximately $50 \%$ of the population aged $\geq 60$ years exhibit $>1.0$ diopter (D) of corneal astigmatism, and $15-29 \%$ have $>1.5$ D. ${ }^{2}$ If their astigmatism is not corrected at the time of cataract surgery, these patients will require spectacle correction postoperatively. Toric intraocular lenses (IOLS) have been developed to help these patients achieve postoperative spectacle independence. In order to reach this goal, it is incumbent upon the surgeon to accurately calculate the power and axis of the toric IOL preoperatively and then to align the IOL along the correct axis at the time of implantation. For each degree the IOL is misaligned there is a loss of scalar astigmatic correction of about 3.5\%. ${ }^{3}$ Misalignment by 30 degrees completely negates the astigmatic correction; postoperatively, the same magnitude of astigmatism will be aligned at a different axis. ${ }^{4}$ Alpins studied the effect of misalignment of astigmatism treatment on the flattening index and found that the loss of effect at 30 degrees is $50 \%$, and is $100 \%$ at 45 degrees. ${ }^{5}$ Similarly, Tognetto et al. found that IOL rotations of $<10$ degrees induced only small amounts of image-quality decay; however, rotations $>30$ degrees reduced the image quality by $50 \%$ and 45 degrees of rotation reduced it to a point at which it was the same as no toric correction at all, with the steepest rates of decay seen between 15 and 20 degrees. ${ }^{6}$ Therefore, correct alignment of the toric IOL along the axis of corneal astigmatism is critical to achieving patient satisfaction.

Historically, the cornea has been marked preoperatively with ink to provide a landmark for toric IOL alignment; however, conventional manual marking methods such as bubble, pendular, or tonometer markers are prone to error due to smudging or dissolution of ink, excessively broad or imprecise marks due to Bell's phenomenon or the cyclotorsion effect, and uncorrected parallax. ${ }^{7}$ Automated systems based on image-guided registration have the potential to remove many of these sources of error. ${ }^{8}$ This technology requires a preoperative, high-definition image which is linked to keratometric data. Intraoperative image analysis then correlates iris landmarks, or conjunctival vessels and scleral pigmentation, to automatically register the intraoperative image to the preoperative image. ${ }^{9}$ The registered image, linked to preoperative keratometric data, can then display the intended axis for IOL alignment on a graphic overlay. Alternatively, the registered image can be used to guide femtosecond laser marking of the cornea with intrastromal corneal cuts. ${ }^{10}$

A new approach to toric IOL alignment involves the construction of small pointers or capsular marks on the capsular rim during femtosecond laser capsulotomy. IntelliAxis capsular marks for toric IOL alignment (IntelliAxis Refractive Capsulorhexis ${ }^{\mathrm{TM}}{ }^{\text {, LENSAR }}{ }^{\circledast}$, Orlando, FL, USA) have the advantage 
Figure 1: Schematic diagram of capsular mark construction ${ }^{11}$

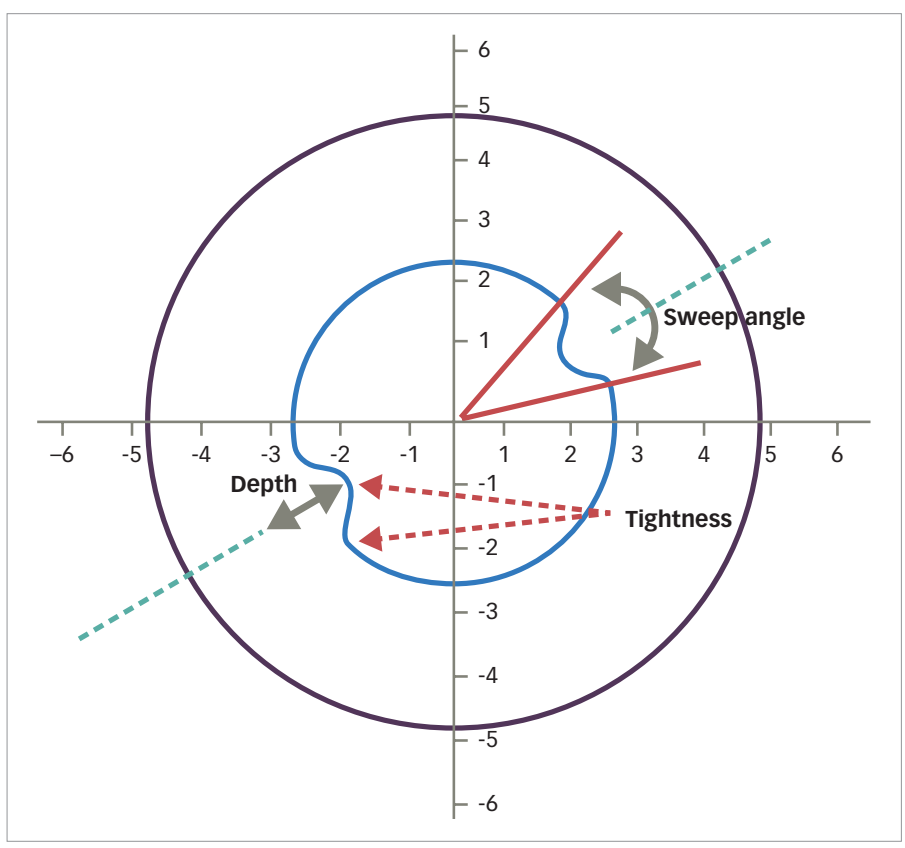

Reused with permission from Valas Teuma et al. ${ }^{11}$

of reducing error due to all of the shortcomings inherent in marking the ocular surface including surgical parallax, and do not impact the strength or extensibility of the capsulotomy. ${ }^{11}$ This paper will review preclinical and clinical data demonstrating the safety and effectiveness of this novel method for toric IOL alignment.

\section{Preclinical study of capsulotomy strength and extensibility}

Safe cataract extraction and IOL implantation require sufficient strength and extensibility of the anterior capsulotomy to avoid anterior capsule tears and capsule rupture. In order to demonstrate the absence of any impact of capsular marks on the integrity of the capsulotomy, Teuma et al. undertook a laboratory study comparing the biomechanical strength of capsulotomies with capsular marks to standard femtosecond laser capsulotomies. ${ }^{11}$ IntelliAxis capsular marks consist of a modification of the standard capsulotomy, in which a pair of tabs or nubs is constructed on the intended axis of toric IOL alignment. Using the LENSAR femtosecond laser system, the axis of implantation can be selected by the surgeon or computed based on corneal and biometric data and the surgeon's specified surgically induced astigmatism.

Figure 1 provides a schematic diagram of the structure of the capsular marks used in Teuma's study. ${ }^{11}$ For this study, the depth of the marks was set at $0.3 \mathrm{~mm}$, the sweep angle was set at 10 degrees, and the curve tightness was set at 0.2. For testing, 36 freshly enucleated porcine eyes were randomly assigned to three treatment cohorts: standard capsulotomy $(n=12)$, capsulotomy with capsular marks for in-line tensile strength testing $(n=12)$, and capsulotomy with capsular marks for orthogonal tensile strength test $(n=12)$. The capsulotomy diameter was $5 \mathrm{~mm}$; laser parameters were as follows: shot-spacing $=5 \mu \mathrm{m}$, Z-spacing $=20 \mu \mathrm{m}$, energy $=7 \mu \mathrm{J}$, pulse repetition frequency $(\mathrm{PRF})=80 \mathrm{kHz}$. After treatment, the eyes were placed in a concave holder and the cornea was removed. Following iridectomy, the limbus and sclera were trimmed posteriorly to allow full range of motion for
Figure 2: Comparison of capsulotomy break force with and without capsular marks ${ }^{11}$

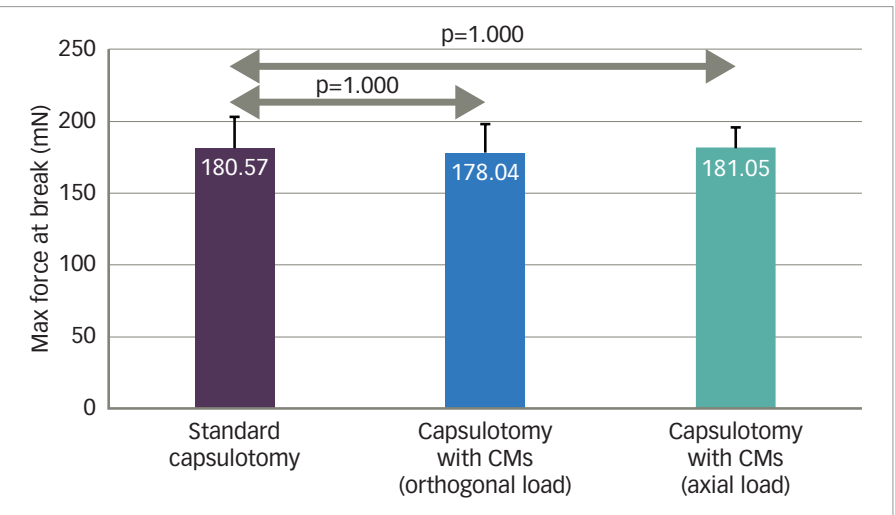

CMs = capsular marks

stretching. The nucleus was then hydro-expressed, and the capsulotomy was stretched as previously described.11

The mean break force for standard capsulotomy, capsulotomy with capsular marks with orthogonal load, and capsulotomy with capsular marks with in-line load were $180.57 \pm 22 \mathrm{mN}, 178.04 \pm 20 \mathrm{mN}$, and $181.05 \pm 15 \mathrm{mN}$, respectively. There was no statistically significant difference in capsular rim strength between the standard capsulotomy and the capsulotomy with capsular marks with tensile force vectors oriented orthogonally $(p=1.000)$ or in-line $(p=1.000)$ (Figure 2$)$. The mean extensibility at the point of rupture for standard capsulotomy, capsulotomy with capsular marks with orthogonal load, and with in-line load were $6.47 \pm 0.33 \mathrm{~mm}$, $6.49 \pm 0.45 \mathrm{~mm}$, and $6.3 \pm 0.47 \mathrm{~mm}$, respectively. Again, there was no statistically significant difference in capsular rim extensibility between the standard capsulotomy and the capsulotomy with capsular marks for tensile force vectors, oriented orthogonally $(p=1.000)$ or in-line $(p=0.960)$ (Figure 3). Thus, the results of this study demonstrate that capsulotomies with capsular marks are equivalent in tensile strength and extensibility to standard laser capsulotomies. ${ }^{11}$

\section{Clinical studies of IntelliAxis capsular marks for toric intraocular lens alignment}

Even small errors in the alignment of toric IOLs can increase residual refractive astigmatism. Automated iris registration for compensation of cyclotorsion may improve accuracy and provide superior outcomes; however, with corneal marking, parallax error may result in IOL misalignment of approximately 2-5 degrees. ${ }^{12}$ With IntelliAxis capsular marks, concern regarding parallax is eliminated because the plane of the anterior capsule is so close to the plane of the IOL. The advantages of the IntelliAxis Refractive Capsulorhexis for toric IOL alignment have been demonstrated in several clinical reports. For example, Visco has reported a retrospective study evaluating visual and refractive outcomes following toric IOL implantation using iris registration-guided femtosecond laser-assisted capsular marks. ${ }^{13}$ The study included 60 eyes that had undergone femtosecond laser-assisted cataract surgery and toric IOL implantation. Results showed reduction in astigmatism from mean keratometric cylinder of $2.11 \mathrm{D}$ preoperatively to refractive cylinder of $0.15 \mathrm{D}$ postoperatively $(p<0.001)$. Fifty-six percent of eyes had no residual astigmatism and $98 \%$ of eyes achieved $\leq 0.5 \mathrm{D}$ residual refractive astigmatism. 
Figure 3: Comparison of capsulotomy extensibility with and without capsular marks ${ }^{11}$

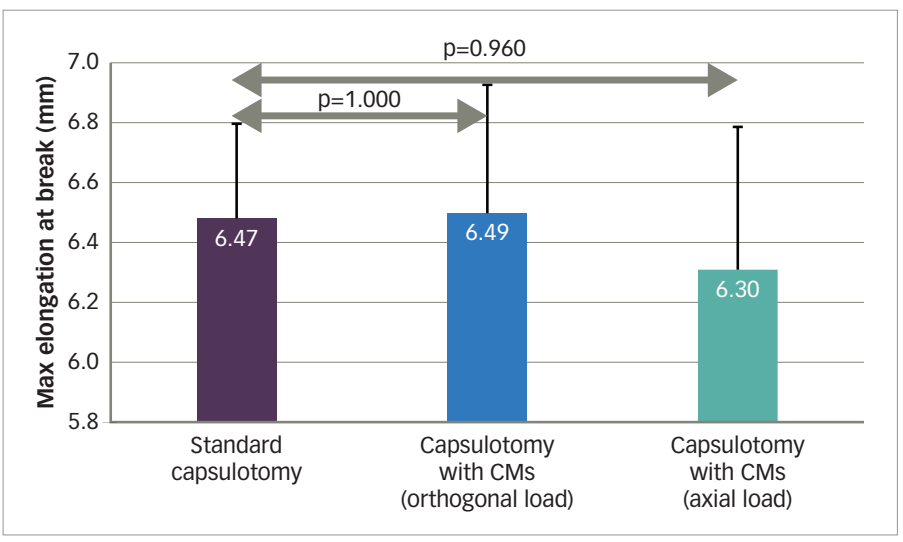

CMs = capsular marks. Reused with permission from valas Teuma et al. ${ }^{11}$

O'Neill et al. reported a prospective case series including 30 eyes of 30 patients with visually significant cataracts and regular astigmatism ranging from 1.5-3.5 D. ${ }^{14}$ All eyes received a toric IOL. Preoperative iris registration was used in conjunction with the LENSAR femtosecond laser to create capsulotomy marks on the intended axis, helping facilitate toric IOL placement. Eyes with concomitant active ocular disease such as diabetic retinopathy, macular degeneration, and any previous ocular surgery were excluded. Patients were examined 1, 7, and 30 days after cataract surgery to assess capsulotomy marks, toric IOL axis, and visual and refractive outcomes. Eighteen eyes completed 1-month follow-up and were included in the study. Mean age was 70.8 years, mean preoperative best-corrected distance visual acuity was $\log$ MAR 0.28 , and mean preoperative keratometric cylinder was $1.98 \mathrm{D}$.

Postoperatively, the capsular toric marks were visible in $88 \%$ of eyes at the 1-day postoperative visits, $50 \%$ at the 1 -week visit, and $24 \%$ at the 1 -month visit. Mean difference between intended toric mark axis and measured toric mark axis at postoperative day 1 was 1.8 degrees and at 1 month it was 0.75 degrees. Mean postoperative uncorrected distance visual acuity was IogMAR 0.1. One eye underwent IOL repositioning at 1 week, at which time the capsular marks were still visible and the IOL was successfully realigned with the original capsular marks. No capsule complications were noted at any time during the study. ${ }^{14}$ The authors concluded that iris-registered femtosecond laser-assisted anterior capsule marks provide a safe, accurate, and effective method for toric IOL alignment at the time of cataract surgery. They also noted that this technique may provide an easily identifiable axis mark postoperatively, that can be used to quickly confirm the toric IOL axis and help with repositioning, if necessary.

In another study, Mckee reported on 21 eyes that had undergone femtosecond laser-assisted cataract surgery and toric IOL implantation for the correction of pre-existing astigmatism. ${ }^{15}$ The Cassini topographer's iris-registration data (Cassini Technologies, The Hague, The Netherlands) were transmitted wirelessly to the LENSAR laser for cyclorotation compensation, and then matched to the laser's intraoperative iris registration to place capsular marks at the intended axis to guide toric IOL alignment. Mean preoperative keratometric cylinder of $1.54 \mathrm{D}$ was reduced to mean $0.32 \mathrm{D}$ residual refractive cylinder. Eighty-one percent of eyes had $\leq 0.5 \mathrm{D}$ postoperative refractive astigmatism and $76.5 \%$ of eyes demonstrated uncorrected visual acuity of 20/25 or better. The author concluded that capsular marks on the anterior capsulotomy rim based on iris registration are effective in aiding the alignment of toric IOL at the desired axis, thus reducing astigmatism.

In a study presented at the 37th Congress of the European Society of Cataract and Refractive Surgeons (ESCRS), Visco, Hill and McKee demonstrated that iris registration-guided femtosecond laser-assisted capsular marks are safe and effective in accurately guiding toric IOL alignment to its intended axis of implantation. ${ }^{16}$ This prospective, multicenter study enrolled 31 eyes of 25 patients with stable regular keratometric cylinder $\geq 0.50 \mathrm{D}$, vector difference of $\leq 0.50 \mathrm{D}$ in anterior corneal astigmatism as measured by the Cassini Corneal Analyzer (Cassini Technologies BV, The Hague, Netherlands) compared with the Zeiss IOLMaster 700 (Carl Zeiss Meditec AG, Jena, Germany) or the Lenstar 900 (Haag-Streit USA, Mason, OH, USA), and predicted residual refractive astigmatism $\leq 0.50$ D. All eyes underwent femtosecond laser-assisted cataract surgery followed by toric IOL implantation. Alignment of toric IOLs was guided by capsular marks integrated into the femtosecond laser capsulotomy. Mean preoperative corneal astigmatism of $2.06 \mathrm{D}$ was reduced to mean postoperative residual refractive astigmatism of $0.11 \mathrm{D}$. All eyes demonstrated residual refractive astigmatism $\leq 0.50 \mathrm{D}, 71.0 \%$ of eyes reported no astigmatism, $12.9 \% \mathrm{had}$ $0.25 \mathrm{D}$, and $16.1 \%$ had $0.50 \mathrm{D}$. The authors concluded that the excellent postoperative refractive outcomes obtained in this study were potentially due to the precise alignment of toric IOL guided by iris fingerprinting that compensates for cyclotorsion.

Warren E Hill, MD, medical director of East Valley Ophthalmology in Mesa, AZ, USA, commented on his experience during this study with the IntelliAxis Refractive Capsulorhexis: "The IntelliAxis Refractive Capsulorhexis solves the problem of toric IOL alignment. Accurate iris registration-guided laser markings within the capsulotomy lay directly on the anterior surface of the IOL. IntelliAxis has converted a problematic aspect of the toric IOL surgery into a non-issue." ${ }^{17}$

In further demonstration of the effectiveness of capsular marks for toric IOL alignment, Jackson et al. compared the astigmatic outcomes of toric IOL implantation obtained using iris registration guided femtosecond laser capsular marks with outcomes using femtosecond steep axis intrastromal corneal marks during cataract surgery. ${ }^{18}$ The baseline characteristics of the two groups were well balanced (Table 1). Postoperative refractive astigmatism was significantly reduced from preoperative keratometric astigmatism in both groups (Figure 4). The authors found that iris registration with the LENSAR femtosecond laser effectively compensates for cyclotorsion and facilitates accurate correction of pre-existing corneal astigmatism. Both corneal (IntelliAxis-C) and capsular (IntelliAxis-L) marks provided excellent guidance for toric IOL alignment. There was an advantage for 20/20 or better uncorrected postoperative visual acuity and $\leq 0.25 \mathrm{D}$ residual refractive cylinder with capsular marks. The authors also noted that the capsular marks facilitated toric IOL alignment by eliminating parallax.

Stephenson utilized adjunctive intraoperative aberrometry (The ORA ${ }^{\mathrm{TM}}$ System, Alcon, Fort Worth, TX, USA) for toric IOL implantation guided by iris registration and femtosecond laser-assisted capsular marks. ${ }^{19}$ In this retrospective analysis, 54 eyes that had undergone femtosecond laser assisted cataract surgery followed by toric IOL implantation with the 
Table 1: Baseline characteristics in comparative study ${ }^{18}$

\begin{tabular}{|l|l|l|}
\hline & $\begin{array}{l}\text { Group 1 (Cassini with } \\
\text { IntelliAxis-C) }\end{array}$ & $\begin{array}{l}\text { Group 2 (Pentacam with } \\
\text { IntelliAxis-L) }\end{array}$ \\
\hline Number & 30 eyes of 20 patients & 20 eyes of 15 patients \\
\hline Age (years) & $61.7 \pm 8.5(42-78)$ & $61.1 \pm 7.9$ years (41-73) \\
\hline Gender & 15 male & 4 male \\
& 5 female & 11 female \\
\hline OD:OS & $14: 16$ & $12: 8$ \\
\hline Preoperative $\Delta K$ & $1.59 \pm 0.59 \mathrm{D}(0.61-2.84)$ & $1.66 \pm 0.77(0.78-3.19)$ \\
\cline { 2 - 3 } & $\mathrm{P}=0.713$ & \\
\hline
\end{tabular}

Figure 4: Preoperative keratometric astigmatism compared with postoperative refractive astigmatism ${ }^{18}$

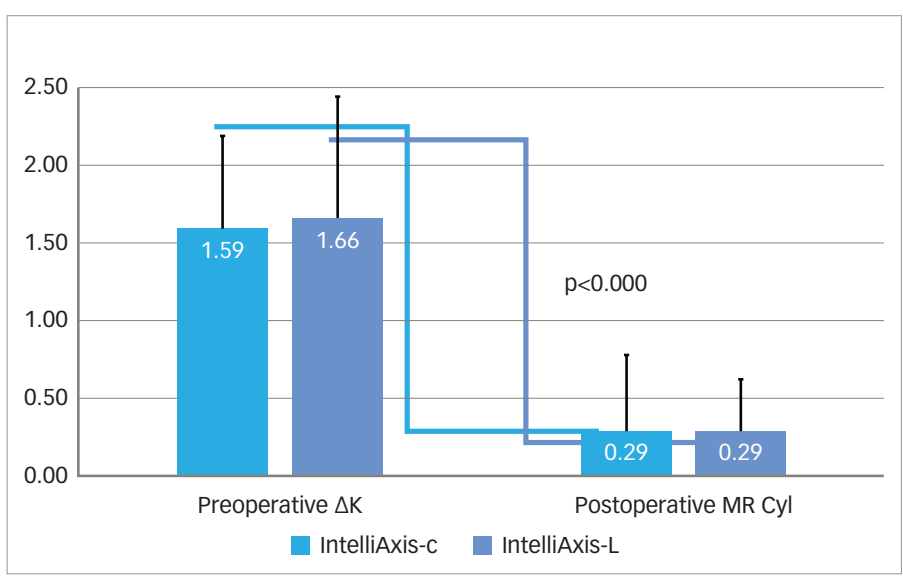

$C y l=$ cylinder; $M R=$ manifest refraction. Reused with permission from Jackson et al..$^{18}$

TRULIGN Toric IOL (Bausch + Lomb, Rochester, NY, USA) were examined at 4-6 weeks postoperative. Axis alignment was guided by iris registration and femtosecond laser capsular marks and confirmed with intraoperative aberrometry. Mean preoperative corneal astigmatism measuring 1.01 D was reduced to mean residual refractive astigmatism of 0.11 D. A total of $94.6 \%$ of eyes exhibited $\leq 0.5 \mathrm{D}$ postoperative refractive cylinder. Uncorrected postoperative visual acuity was $20 / 30$ or better in $97.3 \%$ of eyes.

Chan et al. compared a markerless digital overlay to laser-assisted anterior capsule marks for toric IOL alignment. ${ }^{20}$ Thirty-four eyes with preoperative corneal astigmatism $\geq 1.0 \mathrm{D}$ underwent phacoemulsification with toric IOL implantation. Of these, 18 eyes underwent alignment based on a markerless computer guidance system (CALLISTO ${ }^{\circledR}$ Eye, Carl Zeiss Meditec, Jena, Germany), while 16 eyes underwent alignment based on laser-assisted, anterior capsular marks. The main outcome measures were surgically corrected astigmatism (i.e., the difference between preoperative corneal astigmatism and 1-month postoperative manifest refractive astigmatism) and the difference between predicted and achieved residual astigmatism.

The markerless group and the anterior capsular mark group were similar in terms of mean age and preoperative corneal astigmatism. The mean postoperative manifest refractive astigmatism was similar in the two groups: $0.40 \pm 0.32 \mathrm{D}$ in the markerless group and $0.36 \pm 0.35 \mathrm{D}$ in the capsule marks group ( $p=0.73$ ). The mean surgically corrected astigmatism was also similar:
Figure 5: Cumulative frequency distribution of postoperative refractive astigmatism ${ }^{21}$

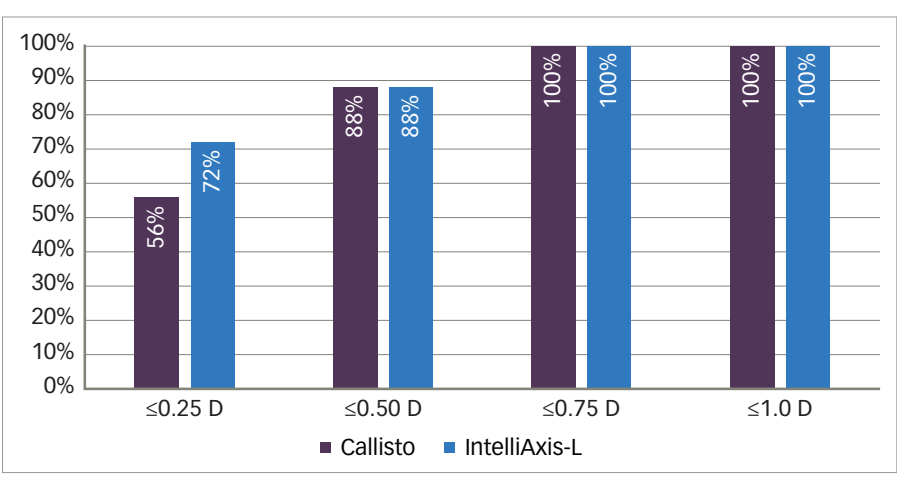

$D=$ diopters

$1.23 \pm 0.61 \mathrm{D}$ in the markerless group and $1.45 \pm 1.11 \mathrm{D}$ in the capsule marks group $(p=0.43)$. For the markerless group, there was a significant difference in mean predicted versus achieved residual astigmatism, $0.02 \pm 0.31 \mathrm{D}$ and $0.15 \pm 0.41 \mathrm{D}(\mathrm{p}=0.03)$; however, for the capsule marks group, the mean predicted residual astigmatism $(0.18 \pm 0.23 \mathrm{D})$ was similar to the achieved residual astigmatism $(0.36 \pm 0.35 \mathrm{D})(\mathrm{p}=0.1) \cdot{ }^{20}$ The authors concluded that both markerless and anterior capsular marking techniques effectively guided toric IOL alignment, resulting in significant astigmatism reduction after refractive cataract surgery; however, the anterior capsule marking technique resulted in superior prediction of postoperative residual refractive astigmatism.

Solomon et al. also compared the efficacy of femtosecond laser assisted IntelliAxis capsular marks to intraoperative computer-assisted biometric surgical guidance with markerless registration for the reduction of refractive cylinder during cataract removal and IOL implantation. ${ }^{21}$ Twenty-four patients underwent toric IOL implantation following phacoemulsification, guided by either of the two methods. Mean preoperative corneal astigmatism was effectively reduced in both groups, from $1.52 \mathrm{D}$ to $0.29 \mathrm{D}$ in the markerless group and from $1.59 \mathrm{D}$ to $0.25 \mathrm{D}$ in the capsular marks group. Eighty-eight percent of eyes in both groups achieved $\leq 0.5 \mathrm{D}$ postoperative refractive cylinder; however, a higher proportion of patients in the capsular marks group achieved residual refractive cylinder $\leq 0.25 \mathrm{D}$ (Figure 5) and a higher proportion of patients in the capsular marks group achieved uncorrected visual acuity 20/20 or better (Figure 6). The authors concluded that femtosecond laser with IntelliAxis-L iris registration and computer-assisted registration with biometric markerless-guidance have comparable outcomes; however, IntelliAxis-L capsular marks were superior at reducing refractive cylinder when positioning toric IOLS.

\section{Conclusion}

Multiple authors have presented outstanding results utilizing IntelliAxis capsular marks for toric IOL alignment. One limitation of these presentations is the omission of calculations of angle of error and correction index. Analysis of postoperative residual refractive astigmatism has demonstrated that $\leq 0.5 \mathrm{D}$ can routinely be achieved in over $80 \%$ of eyes. In addition, studies demonstrate an advantage for capsular marks over other technologies in the achievement of $\leq 0.25 \mathrm{D}$ residual refractive astigmatism and 20/20 or better uncorrected visual acuity. Prediction of residual refractive astigmatism also appears to be superior with capsular marks. 
Figure 6: Postoperative uncorrected distance visual acuity ${ }^{21}$

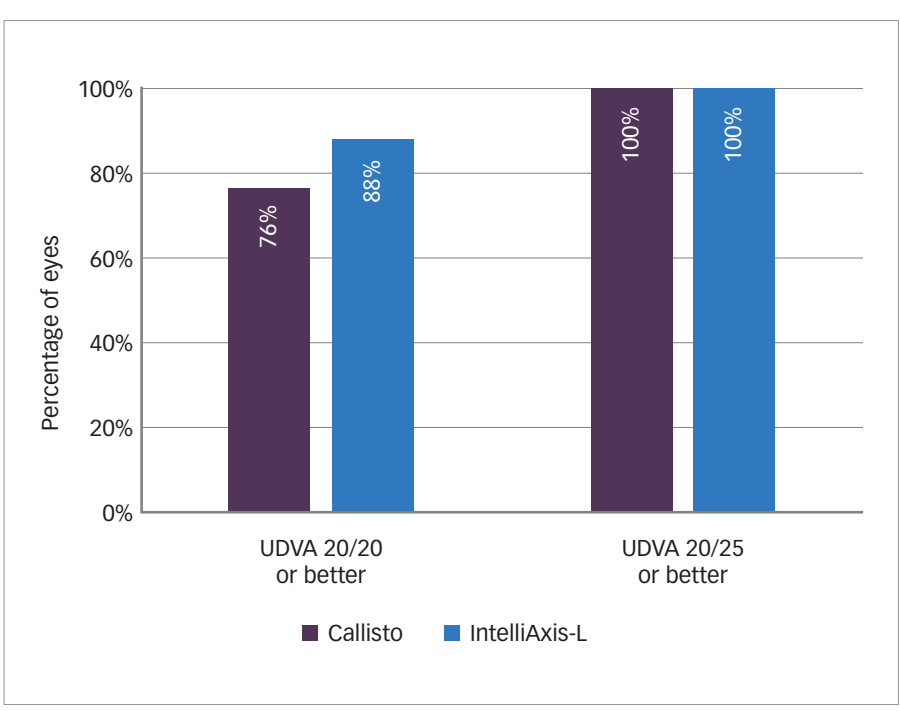

UDVA = uncorrected distance visual acuity.
Iris registration and image-guided femtosecond laser capsular marks avoid many of the pitfalls inherent in older methods of corneal marking, including potential parallax. The utility of capsular marks for postoperative re-alignment of toric IOLs that have rotated also represents a significant advantage of this technology. Authors have noted that capsular marks remain visible during the early postoperative period and can therefore facilitate recognition of rotated toric IOLS and IOL repositioning, if necessary.

Capsular marks for toric IOL alignment represent a noteworthy innovation that was impossible prior to the development and implementation of femtosecond laser assisted cataract surgery. The laboratory demonstration that construction of capsular marks impacts neither the strength nor the extensibility of the capsulotomy provides ample reassurance of the safety of capsular marks. No complications that might have been expected if there were some detriment to capsular resiliency have been reported with this procedure. Accurate alignment of toric IOLs represents a critical step in the reduction of pre-existing corneal astigmatism for patients desiring spectacle independence following cataract surgery. The demonstrated safety and effectiveness of IntelliAxis Refractive Capsulorhexis provides the clinical foundation for improved postoperative results and enhanced patient satisfaction. $\square$
1. Mingo-Botin D, Munoz-Negrete FJ, Won Kim HR, et al. Comparison of toric intraocular lenses and peripheral corneal relaxing incisions to treat astigmatism during cataract surgery. I Cataract Refract Surg 2010:36:1700-8.

2. Hoffmann PC, Hutz WW. Analysis of biometry and prevalence data for corneal astigmatism in 23,239 eyes. J Cataract Refract Surg. 2010;36:1479-85.

3. Ma JJ, Tseng SS. Simple method for accurate alignment in toric phakic and aphakic intraocular lens implantation. $J$ Cataract Refract Surg. 2008;34:1631-6.

4. Poll JT, Wang L, Koch DD, Weikert MP. Correction of astigmatism during cataract surgery: toric intraocular lens compared to peripheral corneal relaxing incisions. J Refract Surg. 2011:27:165-71.

5. Alpins NA. Vector analysis of astigmatism changes by flattening, steepening, and torque. J Cataract Refract Surg. 1997;23: 1503-14.

6. Tognetto D, Perrotta AA, Bauci F, et al. Quality of images with toric intraocular lenses. J Cataract Refract Surg. 2018;44:376-81.

7. Cha D, Kang SY, Kim SH, et al. New axis-marking method for a toric intraocular lens: mapping method. J Refract surg. 2011:27:375-9.

8. Osher RH. Iris fingerprinting: new method for improving accuracy in toric lens orientation. J Cataract Refract Surg. 2010;36:351-2.

9. Kaur M, Shaikh F, Falera R, Titiyal JS. Optimizing outcomes with toric intraocular lenses. Indian J Ophthalmol. 2017:65:1301-13.

10. Montes de Oca I, Kim EJ, Wang L, et al. Accuracy of toric intraocular lens axis alignment using a 3-dimensional computer-guided visualization system. J Cataract Refract surg. 2016;42:550-5

11. Teuma EV, Gray G, Bedi R, Packer M. Femtosecond laser-assisted capsulotomy with capsular marks for toric IOL alignment: comparison of tensile strength with standard femtosecond laser capsulotomy. J Cataract Refract Surg. 2019;45:1177-82

12. Venkataraman A, Kalpana. Visual outcome and rotational stability of open loop toric intraocular lens implantation in Indian eyes. Indian J Ophthalmol. 2013;61:626-9.

13. Visco DM. IRIS registration-guided femtosecond laser-assisted capsular marks to guide toric iol alignment during cataract surgery. Presented at: American Society of Cataract and Refractive Surgery Annual Meeting, San Diego, May 1-5, 2019. Available at: http://ascrs.org/resources/abstracts/iris-registrationguided-femtosecond-laser-assisted-capsular-marks-guide-toriciol-alignment-during (accessed July 11, 2019).

14. O'Neill BP, Diakonis V, Weinstock RJ. Accuracy and stability of iris registered femtosecond laser-assisted anterior capsule axis markings for Toric IOL placement. American Society of Cataract and Refractive Surgery Annual Meeting, San Diego, May 1-5, 2019 Available at: http://ascrs.org/resources/abstracts/accuracy-andstability-risis-registered-femtosecond-laser-assisted-anteriorcapsule-axis-markings (accessed July 11, 2019).

15. McKee $\mathrm{Y}$. Barrett integrated $\mathrm{K}$ method for Toric IOL calculations and iris registration guided femtosecond assisted capsular marks for Toric IOL alignment. American Society of Cataract and Refractive Surgery Annual Meeting, San Diego, May 1-5, 2019. Available at: http://ascrs.org/resources/abstracts/barrettintegrated-k-method-toric-iol-calculations-and-iris-registrationguided-femtosecond-assisted (accessed July 11, 2019).

16. Visco D, Hill W, McKee Y. Safety and effectiveness of a novel femtosecond laser-assisted capsular marking system for the alignment of toric IOLs: a prospective study. Presented at: 37 th Congress of the European Society of Cataract and Refractive Surgeons (ESCRS), Paris, France, September 14-18, 2019. Abstract available at: www.escrs.org/paris2019/programme/free-papersdetails.asp? id $=34306 \& d a y=0$ (accessed October 30 , 2019).

17. Personal Communication. Warren E Hill, MD.

18. Jackson MA, Edmiston A. Toric IOL alignment: femtosecond laser-assisted capsular marks versus intrastromal corneal marks and intraoperative aberrometry. American Society of Cataract and Refractive Surgery Annual Meeting, San Diego, May 1-5, 2019. Available at: http://ascrs.org/resources/abstracts/toric-iolalignment-femtosecond-laser-assisted-capsular-marks-versusintrastromal-corneal-marks-and (accessed July 11, 2019).

19. Stephenson PDG. Femtosecond laser-assisted capsular marks and ORA to guide Toric IOL alignment during cataract surgery. American Society of Cataract and Refractive Surgery Annual Meeting, San Diego, May 1-5, 2019. Available at: http://ascrs. org/resources/abstracts/femtosecond-laser-assisted-capsularmarks-and-ora-guide-toric-iol-alignment-during-cataract-surgery (accessed July 11, 2019)

20. Chan PS, Uy H. Comparison of markerless versus laser-assisted anterior capsule marking for Toric IOL alignment. American Society of Cataract and Refractive Surgery Annual Meeting, San Diego, May 1-5, 2019. Available at: http://ascrs.org/ resources/abstracts/comparison-markerless\%C2\%A0versusresources/abstracts/comparison-markerless\%C2\%AOversus(accessed July 11, 2019).

21. Solomon JD, O'Neill B. Comparison of the efficacy of femtosecond laser-assisted intelliaxis-I to intraoperative computer-assisted biometric surgical guidance with markerless registration for Toric intraocular lens placement. American Society of Cataract and Refractive Surgery Annual Meeting, San Diego, May 1-5, 2019. Available at: http://ascrs.org/resources/abstracts/comparisonefficacy-femtosecond-laser-assisted-intelliaxis-l-intraoperativecomputer-assisted (accessed July 11, 2019). 